\title{
Different prognosis by subtype in the early mucinous breast cancer: a SEER population-based analysis
}

\author{
Weibin Lian ${ }^{1}$, Juanjuan Zheng ${ }^{2}$, Debo Chen ${ }^{1}$ \\ ${ }^{1}$ Department of Breast Surgery, The Affiliated Quanzhou First Hospital of Fujian Medical University, Quanzhou, China; ${ }^{2}$ Department of Medical- \\ Records, The Affiliated Quanzhou First Hospital of Fujian Medical University, Quanzhou, China \\ Contributions: (I) Conception and design: W Lian; (II) Administrative support: W Lian, D Chen; (III) Provision of study materials or patients: W \\ Lian; (IV) Collection and assembly of data: W Lian; (V) Data analysis and interpretation: J Zheng; (VI) Manuscript writing: All authors; (VII) Final \\ approval of manuscript: All authors. \\ Correspondence to: Weibin Lian; Debo Chen. Department of Breast Surgery, The Affiliated Quanzhou First Hospital of Fujian Medical University, \\ No. 250 East Street, Licheng District, Quanzhou 362000, China. Email: weibinlian@fjmu.edu.cn; deboqz@163.com.
}

Background: Our study aims to investigate the clinicopathological characteristics and survival outcomes of mucinous breast cancer (MBC), and explore the effect of histology type on the breast cancer-specific survival (BCSS) by different subtypes.

Methods: we identified 7,083 patients who were diagnosed with MBC and 248,751 with infiltrating ductal carcinoma (IDC) by using the Surveillance, Epidemiology and End Results (SEER) database. The propensity score matching was used to match baseline characteristics among MBC and IDC, and multivariable cox proportional hazards models were used to analyze the relationship between histology type stratified by subtype and BCSS.

Results: MBC patients were associated with a fewer nodal involvement, lower grade, earlier stage, more estrogen receptor (ER) or progesterone receptor (PR) positive, and more favorable prognosis compared to the overall IDC population. After 1:1 matching of MBC with IDC by other factors, we found that MBC patients presented better prognosis than the Matched IDC for BCSS. Analysis among ER+PR+ subgroup revealed that MBC patients was significantly better than that Matched IDC patients for BCSS (HR $=0.78$, 95\% CI, 0.63-0.96). However, the survival analysis in the ER+PR- or ER-PR- subgroups suggested that no significant difference was seen between MBC patients and matched IDC patients for BCSS.

Conclusions: Our findings support that $\mathrm{MBC}$ seems to be an independent factor for the better prognosis for breast cancer patients with $\mathrm{ER}+\mathrm{PR}+$ breast cancer but not in those with ER+PR- or ER-PR- disease.

Keywords: Mucinous breast cancer (MBC); survival; subtype

Submitted Feb 27, 2020. Accepted for publication Aug 28, 2020.

doi: $10.21037 /$ tcr-20-1237

View this article at: http://dx.doi.org/10.21037/tcr-20-1237

\section{Introduction}

Mucinous breast cancer (MBC) is a rare histological type, which was reported to account for $1 \%$ to $7 \%$ of all invasive breast cancer (1-3). Previous studies suggested that MBC differs from other histological breast cancer types and has favorable prognosis (90\% overall survival and $96 \%$ breast cancer-special survival at 10 years) $(4,5)$. MBC was associated with a smaller tumor size, lower rates of nodal involvement, a high expression of hormone receptors (HR) and more human epidermal growth factor receptor 2 (HER2)-negative than infiltrating ductal carcinoma (IDC) $(2,6)$. What is confusing is that whether the good prognosis of $\mathrm{MBC}$ is due to good biological behavior or just as an independent prognostic factor. However, some previous reports have biased results due to the limitation of study population and insufficient follow-up time. Additionally, most of the current treatment guidelines for MBC are 
extrapolated from IDC, and these disputes may affect MBC therapeutic strategies. Therefore, this may help us interpret the clinicopathological characteristics and prognosis of MBC better base on a larger population and longer followup time, and develop more accurate therapeutic strategies for MBC patients. Furthermore, the NCCN guideline (V2. 2019) recommends using the estrogen receptor (ER) and progesterone receptor (PR) status as the important influencing factor for therapeutic strategies. As far as we know, few studies have focussed on the effect of histology type (MBC versus IDC) for prognosis by subtype in women with breast cancer. We conducted a subgroup analysis to investigate the effect of histology type (MBC versus IDC) on the BCSS by different subtypes of breast cancer with a large study population by using the Surveillance, Epidemiology and End Results (SEER) database. We present the following article in accordance with the STROBE reporting checklist (available at http://dx.doi. org/10.21037/tcr-20-1237).

\section{Methods}

\section{Data sources}

Data were obtained from the SEER cancer database, which consists of 18 cancer registries across the United States and is regarded as nationally representative. We selected women age 18 or older between 2004 and 2012 who pathologic confirmation of non-metastatic unilateral IDC (International Classification of Diseases for Oncology code, ICD-0-3 8500/3) and MBC (MBC, ICD-0-3 8480/3). Those who either had had a previous diagnosis of malignant tumor or who without surgically treated were excluded in our study. All patients who were missing ER or PR status and survived less than one month were also excluded. Finally, we identified 255,834 patients, among who 7,083 were diagnosed with MBC and 248,751 with IDC. The study was conducted in accordance with the Declaration of Helsinki (as revised in 2013). Our study was approved by the Affiliated Quanzhou First Hospital of Fujian Medical University Ethics committee (No. 002965).

\section{Study variables}

SEER*Stat version 8.3.5 was used to obtain the demographic, pathological characteristics and therapy information on the following factors: year of diagnosis, age at diagnosis, race/ethnicity, American Joint Committee on
Cancer (AJCC) TNM stage, histologic grade, the status of ER, PR and human HER2, surgery, chemotherapy, radiation, and breast cancer-specific survival (BCSS). All of variables were regrouped and given in Table 1 .

\section{Statistical analyses}

Analyses were conducted using Stata software (version 15.1 ) and assumed a 2-tailed $\alpha=0.05$. The breast cancerspecial survival (BCSS), which was defined as the time from diagnosis to the death caused by breast cancer, was considered as the outcomes of our study. The followup deadline was December 31, 2015. We compared demographics and clinicopathological characteristics between MBC and IDC using the Chi-square test or Fisher exact test. The Kaplan-Meier plot was used to analyze survival curves. Univariate and multivariate Cox proportional hazard models were applied to assess the relationship between these factors and BCSS.

In order to resolve the differences in baseline characteristics across groups, we used the propensity score matching method to match each MBC patient to one IDC patient based on these factors, and tested the matching quality for the balance. Multivariable Cox proportional hazards regression was developed to estimate the HR and 95\% CI for the relationship between histology subtype (MBC versus IDC) and BCSS within each subgroup (according to ER or PR status). Adjustments were then carried out on multivariate analyses which included patient's age, race, stage, grade, surgery, chemotherapy and radiation.

\section{Results}

\section{Patient characteristics}

The study enrolled 255,833 patients, and the baseline characteristics for IDC $(n=7,084)$ and MBC $(n=248,750)$ are summarized in Table 1. The differences between the $\mathrm{MBC}$ and IDC groups for all the variables were statistically significant $(\mathrm{P}=0.000)$. MBC patients were associated with a fewer negative lymph nodes (for N0: $90.27 \%$ vs. 67.48\%), lower grade (for grade 1: $54.3 \%$ vs. 19.2\%), earlier stage (for stage I: $65.35 \%$ vs. $50.85 \%$ ), and more ER or PR positive (98.01\% vs. $77.59 \%, 88.31 \%$ vs. $67.23 \%$, respectively) compared with IDC. Regarding treatment type, a higher percentage of $\mathrm{MBC}$ patients underwent breast-conserving surgery $(67.41 \%$ vs. $59.82 \%)$ and a lower percentage of MBC patients accept chemotherapy (14.98\% vs. 45.64\%) 
Table 1 Patient and tumor characteristics

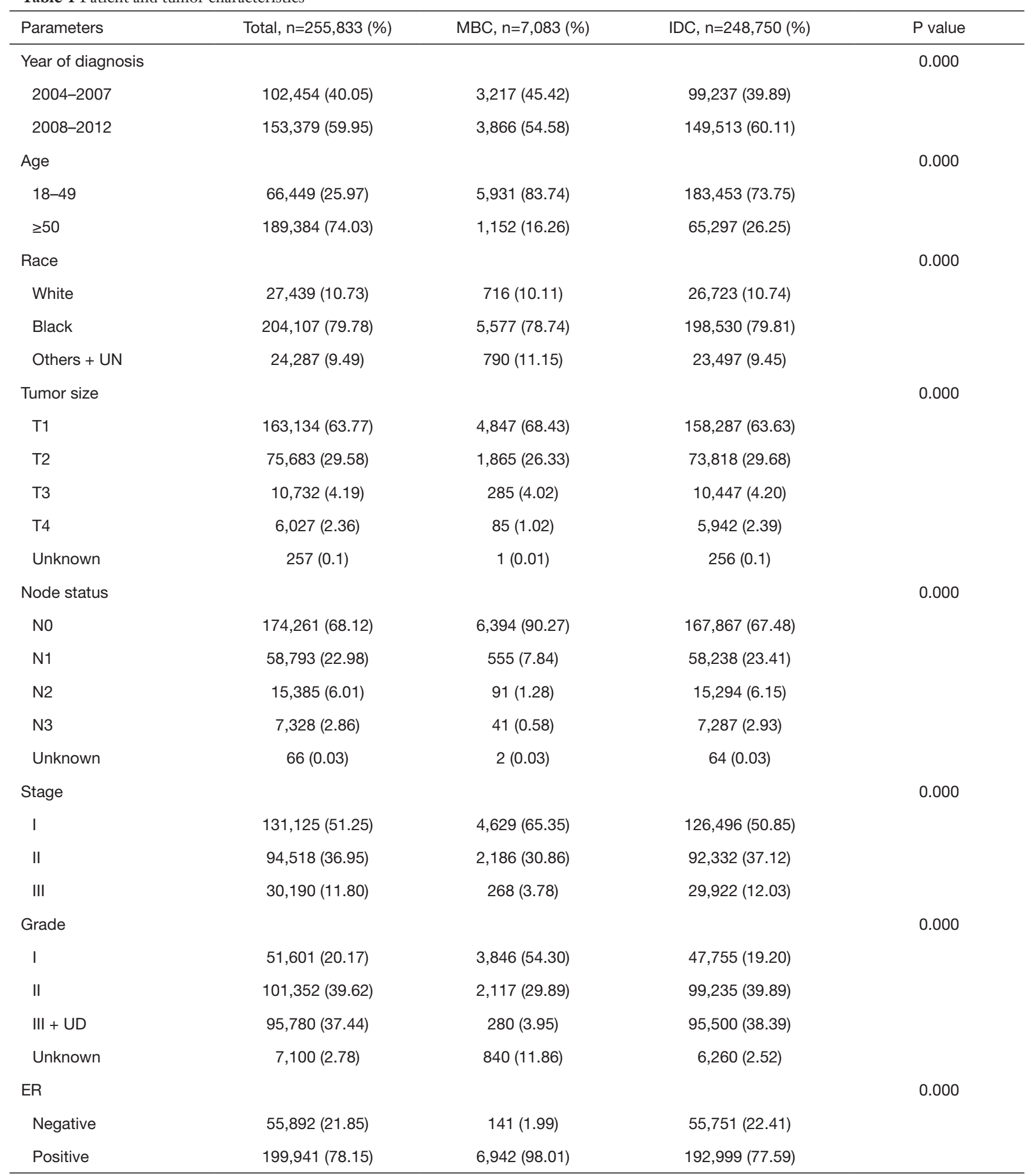

Table 1 (continued) 
Table 1 (continued)

\begin{tabular}{|c|c|c|c|c|}
\hline Parameters & Total, n=255,833 (\%) & $\mathrm{MBC}, \mathrm{n}=7,083(\%)$ & IDC, $n=248,750(\%)$ & $P$ value \\
\hline Negative & 82,335 (32.18) & $828(11.69)$ & $81,507(32.77)$ & \\
\hline Positive & $173,498(67.82)$ & $6,255(88.31)$ & $167,243(67.23)$ & \\
\hline HER2 & & & & 0.000 \\
\hline Positive & $14,368(5.62)$ & $125(1.76)$ & 14,243 (5.73) & \\
\hline Uncertain & $2,023(0.79)$ & $42(0.59)$ & $1,981(0.80)$ & \\
\hline Unknown & $164,292(64.22)$ & $4,833(68.23)$ & $159,459(64.10)$ & \\
\hline Surgery & & & & 0.000 \\
\hline Chemotherapy & & & & 0.000 \\
\hline Yes & $114,580(44.79)$ & $1,061(14.98)$ & $113,519(45.64)$ & \\
\hline No & $141,253(55.21)$ & $6,022(85.02)$ & $135,231(54.36)$ & \\
\hline Radiation & & & & 0.000 \\
\hline Yes & $141,627(55.36)$ & $3,676(51.90)$ & $137,951(55.46)$ & \\
\hline No & $114,206(44.64)$ & 3,407 (48.10) & $110,799(44.54)$ & \\
\hline
\end{tabular}

ER, estrogen receptor; HER2, human epidermal growth factor receptor 2; PR, progesterone receptor; MBC, mucinous breast cancer carcinoma; IDC, infiltrating ductal carcinoma, BCSS, breast cancer-specific survival.

compared with IDC.

\section{BCSS analysis in $M B C$}

Univariate analysis indicated that race, grade, tumor size, node status, stage, grade, ER or PR status, surgery type, chemotherapy and radiation were statistically significant prognostic factors for BCSS (Table 2). With multivariable analysis using the Cox's proportional hazards model, we found that MBC patients with large-sized tumors, node involvement, higher grade, and ER or PR negative were significantly associated with worse BCSS (Table 2). White women with $\mathrm{MBC}$ were significantly more likely to die from breast cancer than other races. Patients who undergoing a mastectomy did not show better BCSS compared with who received breast conservation therapy ( $\mathrm{HR}=0.90,95 \%$ CI, 0.72-1.13). No significant difference was seen between adjuvant chemotherapy or not for BCSS (HR $=0.91,95 \%$ CI, 0.70-1.18).

\section{Survival analysis in matched group}

From the Figure 1, patients with $\mathrm{MBC}$ were associated with better BCSS than the overall IDC population. We obtained a group of 7,083 IDC patients as matched groups through 1:1 (MBC/IDC) propensity score matching method. We found no statistically significant difference in characteristics between MBC and IDC except histologic grade (Table 3). Furthermore, we found that $\mathrm{MBC}$ patients still presented better BCSS than the Matched IDC (Figure 2).

\section{Stratification by molecular subtype}

Multivariable Cox proportional hazards regression was used to describe the association between histology type (MBC and IDC) and BCSS by subtype. After adjusting for age, race, stage, grade, surgery, chemotherapy and radiation, MBC patients in the ER+PR+ subgroup was significantly better than that Matched IDC patients for BCSS (HR $=0.78,95 \% \mathrm{CI}, 0.63-0.96)$. Additionally, the survival 
Table 2 Univariate and multivariate analyses of BCSS with MBC using Cox proportional hazards modeling

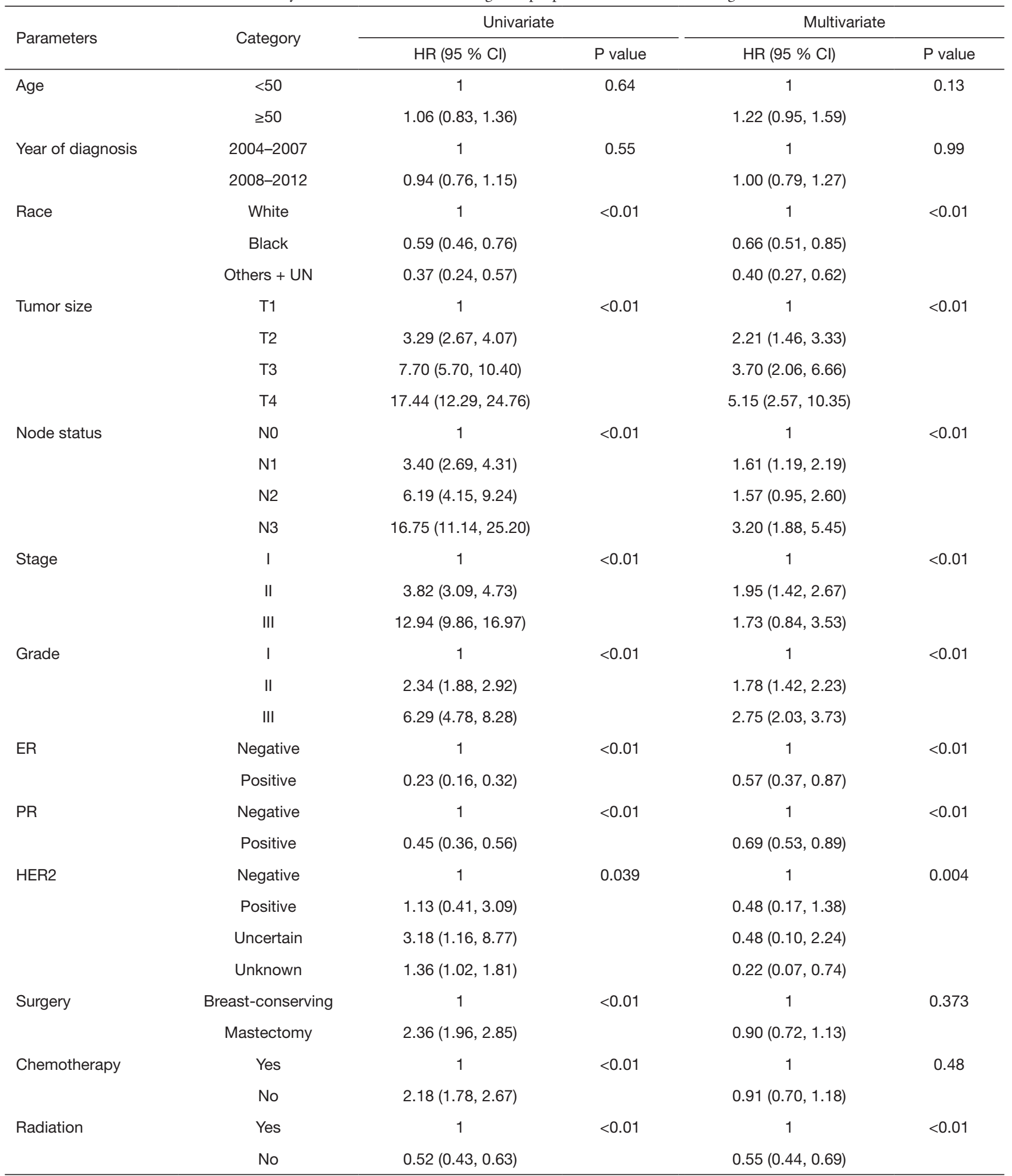

ER, estrogen receptor; HER2, human epidermal growth factor receptor 2; PR, progesterone receptor; MBC, mucinous breast cancer carcinoma; IDC, infiltrating ductal carcinoma; BCSS, breast cancer-specific survival. 


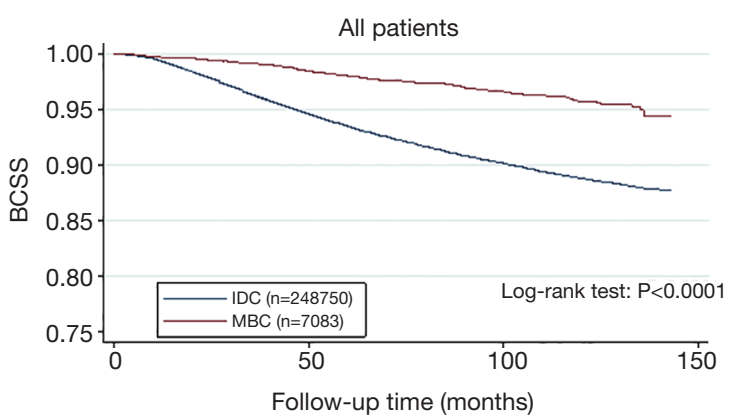

Figure 1 Kaplan-Meier curves by histologic subtypes of breast cancer, MBC versus IDC. MBC, mucinous breast cancer carcinoma; IDC, infiltrating ductal carcinoma, BCSS, breast cancer-specific survival. analysis in the ER+PR- or ER-PR- subgroups suggested that the BCSS of MBC patients was similar to that of Matched IDC patients (Table 4).

\section{Discussion}

Our study aimed to investigated the clinicopathological characteristics and survival outcomes of $\mathrm{MBC}$, and explore the effect of histology type (MBC versus IDC) on the BCSS by subtypes based on a large population. Our findings indicated that $\mathrm{MBC}$ have favorable clinicopathological characteristics, fewer nodal involvement, lower grade, earlier tumor stage and more hormone receptor positive, and are

Table 3 Characteristics of patients by histology type in 1:1 matched, MBC versus IDC

\begin{tabular}{|c|c|c|c|c|}
\hline Parameters & Total, $n=14,166$ & $\mathrm{MBC}, \mathrm{n}=7,083$ & IDC, $n=7,083$ & $P$ value \\
\hline 2004-2007 & 6,405 & 3,217 & 3,188 & \\
\hline 2008-2012 & 7,761 & 3,866 & 3,895 & \\
\hline Age & & & & 1.0000 \\
\hline$\geq 50$ & 11,862 & 5,931 & 5,931 & \\
\hline Race & & & & 0.6552 \\
\hline White & 11,187 & 5,577 & 5,610 & \\
\hline Black & 1,433 & 716 & 717 & \\
\hline $\mathrm{T} 1$ & 9,748 & 4,847 & 4,901 & \\
\hline $\mathrm{T} 2$ & 3,696 & 1,865 & 1,831 & \\
\hline T3 & 528 & 285 & 243 & \\
\hline $\mathrm{T} 4$ & 189 & 85 & 104 & \\
\hline Unknown & 5 & 1 & 4 & \\
\hline Node status & & & & 0.6682 \\
\hline NO & 12,793 & 6,394 & 6,399 & \\
\hline N1 & 1,102 & 555 & 547 & \\
\hline
\end{tabular}

Table 3 (continued) 
Table 3 (continued)

\begin{tabular}{|c|c|c|c|c|}
\hline Parameters & Total, $n=14,166$ & $\mathrm{MBC}, \mathrm{n}=7,083$ & IDC, $n=7,083$ & $P$ value \\
\hline I & 9,307 & 4,629 & 4,678 & \\
\hline II & 4,315 & 2,186 & 2,129 & \\
\hline III & 544 & 268 & 276 & \\
\hline I & 7,604 & 3,846 & 3,758 & \\
\hline II & 4,330 & 2,117 & 2,213 & \\
\hline III + UD & 708 & 280 & 428 & \\
\hline Unknown & 1,524 & 840 & 684 & \\
\hline Positive & 13,872 & 6,942 & 6,930 & \\
\hline PR & & & & 0.8346 \\
\hline Negative & 1,664 & 828 & 836 & \\
\hline Positive & 12,502 & 6,255 & 6,247 & \\
\hline HER2 & & & & 0.9882 \\
\hline Negative & 4,176 & 2,083 & 2,093 & \\
\hline Positive & 245 & 125 & 120 & \\
\hline Uncertain & 84 & 42 & 42 & \\
\hline Yes & 2,107 & 1,061 & 1,046 & \\
\hline No & 12,059 & 6,022 & 6,037 & \\
\hline Radiation & & & & 0.7240 \\
\hline Yes & 7,331 & 3,676 & 3,655 & \\
\hline No & 6,835 & 3,407 & 3,428 & \\
\hline
\end{tabular}

ER, estrogen receptor; HER2, human epidermal growth factor receptor 2; PR, progesterone receptor; MBC, mucinous breast cancer carcinoma; IDC, infiltrating ductal carcinoma, BCSS, breast cancer-specific survival. 
associated with better BCSS for overall IDC. Furthermore, to make sure that baseline balanced in demographic and tumor characteristics across histologic types, we carried out a 1:1 (MBC/IDC) matched case-control analysis using the propensity score matching method to evaluate the survival outcome between MBC and IDC and the subgroup analyses. We found that $\mathrm{MBC}$ presented similar better prognosis than the Matched IDC for BCSS. MBC patients present a better prognosis for those with the $\mathrm{ER}+\mathrm{PR}+$ subtype but not in those with $\mathrm{ER}+\mathrm{PR}-$ or ER-PR- disease.

As the largest study of MBC to date, our study utilizes

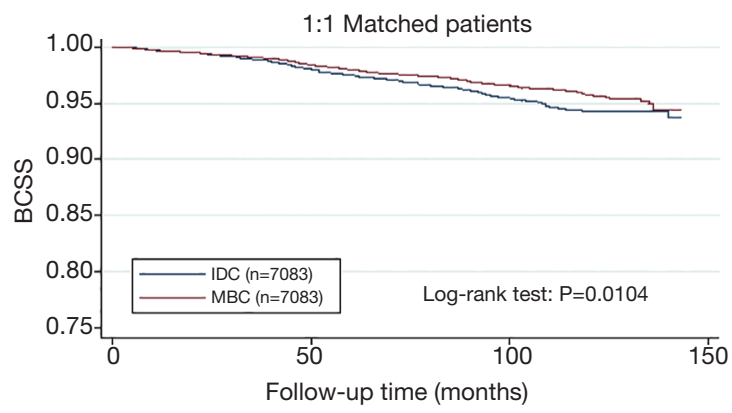

Figure 2 Kaplan-Meier curves for 1:1 matched groups by histology, MBC (matched) versus IDC (matched). MBC, mucinous breast cancer carcinoma; IDC, infiltrating ductal carcinoma; BCSS, breast cancer-specific survival. a large number of SEER datasets to investigate the clinicopathological characteristics. Just as reported in previous studies, the BCSS of MBC was significantly better than IDC, however, this may be mostly due to the fact that the clinicopathological characteristics of $\mathrm{MBC}$ was superior to IDC. The MBC histological type showed lower grade, smaller tumor size, few nodal involvement, earlier stage, higher expression of hormone receptors than IDC. Therefore, propensity score matching was used to match baseline characteristics and ensured these between MBC and IDC groups balanced. After 1:1 matching of MBC with IDC by age, race, tumor size, node status, stage, grade, ER status, PR status, HER2 status, subtype, surgery, chemotherapy and radiation, $\mathrm{MBC}$ showed similarly the better BCSS than Matched IDC. These results imply that the MBC histological type seem to be an independent prognostic factor.

More and more experts recommend molecular subtypes of breast cancer as the important reference factors for therapeutic strategies. Our study focused on subgroup analysis using the ER and PR statuses of MBC and Matched IDC. A multivariate Cox analysis indicated that MBC was an independent predictor of a better BCSS compared with Matched IDC in ER+PR+ group. From the Table 3, we see that the difference whether in chemotherapy or radiotherapy group between these two pathological types was not showed statistically significant. Endocrine therapy

Table 4 Histology type and BCSS by ER and PR status

\begin{tabular}{|c|c|c|c|c|}
\hline Subtype/histology & Patients, N & BCSS, N (\%) & $\mathrm{HR}(95 \% \mathrm{Cl})^{\star}$ & $P$ \\
\hline \multicolumn{5}{|l|}{$\mathrm{ER}+/ \mathrm{PR}+$} \\
\hline IDC & 6,232 & 6,042 (96.9) & 1 & 0.021 \\
\hline MBC & 6,244 & 6,096 (97.6) & $0.78(0.63,0.96)$ & \\
\hline \multicolumn{5}{|l|}{$\mathrm{ER}+/ \mathrm{PR}-$} \\
\hline IDC & 698 & $661(94.7)$ & 1 & 0.538 \\
\hline MBC & 698 & 667 (95.5) & $0.86(0.53,1.39)$ & \\
\hline \multicolumn{5}{|l|}{ ER-/PR+ } \\
\hline IDC & 15 & $13(86.6)$ & 1 & - \\
\hline MBC & 11 & $11(100.0)$ & - & \\
\hline \multicolumn{5}{|l|}{ ER-/PR- } \\
\hline IDC & 138 & $138(100.0)$ & 1 & 0.300 \\
\hline $\mathrm{MBC}$ & 130 & $115(88.4)$ & $0.70(0.36,1.36)$ & \\
\hline
\end{tabular}

*, adjusted for age, race, stage, grade, surgery, chemotherapy, radiation. ER, estrogen receptor; PR, progesterone receptor; MBC, mucinous breast cancer; IDC, infiltrating ductal carcinoma. 
was available in this subtype. Unfortunately, we failed to acquire data about that. We considered that MBC perform better endocrine-responsive than Matched IDC in the $\mathrm{ER}+\mathrm{PR}+$ subtype. However, MBC seem not exhibited a significantly better BCSS than Matched IDC in ER+PR- or ER-PR- subgroups. Several studies had reported that loss of PR was associated with one mechanism for endocrine resistance and resulted for higher risk of relapse $(7,8)$. Furthermore, it has reported that ER+PR- tumors are more likely to have high expression of epidermal growth factor receptor (8-11). Bae et al. has suggested that ER+PRhad comparable poor survival to ER-PR- tumors with invasive ductal carcinoma (8-11). It can be seen that the $\mathrm{ER}+\mathrm{PR}-$ tumors in $\mathrm{MBC}$ should be treated aggressively as same as IDC.

Our research also has several limitations. First, it is a retrospective study and may have some potential selection bias. Then we failed to differentiate mixed mucinous carcinoma and pure mucinous carcinoma. Furthermore, the status of HER2 was not included for further subtype analysis, but HER2 information in the SEER datasets was not available until 2010 and resulted for lacking adequate patients for subgroup analysis. However, our study is the first to incorporate ER and PR status for subgroup analysis in the MBC survival studies. Further studies are needed to focus on its molecular subtypes.

In conclusion, $\mathrm{MBC}$ is a histological subtype that is distinct from IDC with favorable clinicopathological characteristics and prognosis. MBC seems to be an independent factor for the better prognosis with $\mathrm{ER}+\mathrm{PR}+$ breast cancer but not in those with ER+PR- or ER-PRdisease. Therefore, an escalated therapeutic strategy may be considered for ER+PR- or ER-PR- MBC patients.

\section{Acknowledgments}

Funding: This work was supported by grants from the Science and Technology Programme of Quanzhou (2018N073S).

\section{Footnote}

Reporting Checklist: The authors have completed the STROBE reporting checklist. Available at http://dx.doi. org/10.21037/tcr-20-1237

Peer Review File: Available at http://dx.doi.org/10.21037/tcr20-1237
Conflicts of Interest: All authors have completed the ICMJE uniform disclosure form (available at http://dx.doi. org/10.21037/tcr-20-1237). The authors have no conflicts of interest to declare.

Ethical Statement: The authors are accountable for all aspects of the work in ensuring that questions related to the accuracy or integrity of any part of the work are appropriately investigated and resolved. All methods were carried out in accordance with relevant guidelines and regulations. The study was conducted in accordance with the Declaration of Helsinki (as revised in 2013). Our study was approved by the Affiliated Quanzhou First Hospital of Fujian Medical University Ethics committee (No. 002965).

Open Access Statement: This is an Open Access article distributed in accordance with the Creative Commons Attribution-NonCommercial-NoDerivs 4.0 International License (CC BY-NC-ND 4.0), which permits the noncommercial replication and distribution of the article with the strict proviso that no changes or edits are made and the original work is properly cited (including links to both the formal publication through the relevant DOI and the license). See: https://creativecommons.org/licenses/by-nc-nd/4.0/.

\section{References}

1. Anderson WF, Chu KC, Chang S, et al. Comparison of age-specific incidence rate patterns for different histopathologic types of breast carcinoma. Cancer Epidemiol Biomarkers Prev 2004;13:1128-35.

2. Di Saverio S, Gutierrez J, Avisar E. A retrospective review with long term follow up of 11,400 cases of pure mucinous breast carcinoma. Breast Cancer Res Treat 2008;111:541-7.

3. Park S, Koo J, Kim JH, et al. Clinicopathological characteristics of mucinous carcinoma of the breast in Korea: comparison with invasive ductal carcinoma-not otherwise specified. J Korean Med Sci 2010;25:361-8.

4. Fu J, Wu L, Jiang M, et al. Clinical Nomogram for Predicting Survival Outcomes in Early Mucinous Breast Cancer. PLoS One 2016;11:e0164921.

5. Li CI. Risk of mortality by histologic type of breast cancer in the United States. Horm Cancer 2010;1:156-65.

6. Louwman MW, Vriezen M, van Beek MW, et al. Uncommon breast tumors in perspective: incidence, treatment and survival in the Netherlands. Int J Cancer 2007;121:127-35. 
7. Cancello G, Maisonneuve P, Rotmensz N, et al. Progesterone receptor loss identifies Luminal B breast cancer subgroups at higher risk of relapse. Ann Oncol 2013;24:661-8.

8. Cui X, Schiff R, Arpino G, et al. Biology of progesterone receptor loss in breast cancer and its implications for endocrine therapy. J Clin Oncol 2005;23:7721-35.

9. Prat A, Cheang MC, Martín M, et al. Prognostic significance of progesterone receptor-positive tumor cells within immunohistochemically defined luminal A breast cancer. J Clin Oncol 2013;31:203-9.

10. Braun L, Mietzsch F, Seibold P, et al. Intrinsic breast cancer subtypes defined by estrogen receptor signallingprognostic relevance of progesterone receptor loss. Mod Pathol 2013;26:1161-71.

11. Bae SY, Kim S, Lee JH, et al. Poor prognosis of single hormone receptor- positive breast cancer: similar outcome as triple-negative breast cancer. BMC Cancer 2015;15:138.

Cite this article as: Lian W, Zheng J, Chen D. Different prognosis by subtype in the early mucinous breast cancer: a SEER population-based analysis. Transl Cancer Res 2020;9(10):5969-5978. doi: 10.21037/tcr-20-1237 